\begin{tabular}{ll} 
& CC (†) \\
& Esta obra está sob o \\
& direito de Licença \\
& Creative Commons \\
& Atribuição \\
& Internacional. \\
\hline
\end{tabular}

\title{
GAMETERAPIA COMO TECNOLOGIA ASSISTIVA
}

Sandra Maria Ponte ${ }^{1}$

Andrea Marques Vanderlei Fregadolli ${ }^{2}$

Adriana Cavalcante da Silva ${ }^{3}$

Audeluze Maria Araújo Victor De Mendonça Lopes ${ }^{4}$

Elizabeth Calheiros Borges ${ }^{5}$

Isaac Assunção Ferreira ${ }^{6}$

\section{RESUMO}

A gameterapia é considerada um método inovador e/ou motivador no processo de reabilitação dos pacientes que se encontram em processo de tratamento de reabilitação. O estudo buscou discutir cientificamente os vídeos compartilhados pelo sítio YouTube que tratam da gameterapia como tecnologia assistiva, analisando vídeos postados no período entre 2014 a 2019. Trata-se de uma revisão sistemática. Utilizando os descritores estruturados no DeCS e MeSH. O período de coleta dos dados ocorreu em outubro/2019. Adotaram-se como critérios de inclusão tipos, duração e data de upload e, como critérios de exclusão vídeos que não atendem a temática estudada. Os vídeos sinalizaram para um conceito positivo acerca da gameterapia como tecnologia assistiva, pautado em uma visão sistêmica, que procura motivar os pacientes que estão em processo de tratamento e/ou reabilitação na promoção de uma melhor qualidade de vida.

Palavras-chave: Gameterapia. Tecnologia Assistiva. Motivação. Reabilitação.

\footnotetext{
${ }^{1}$ sandrinha.2005@hotmail.com

2 deadoutorado@hotmail.com

3 Adrianacavalcante32@hotmail.com

${ }^{4}$ Del.fest@hotmail.com

${ }^{5}$ bethcalheirosborges@gmail.com

6 isaacassuncaoferreia@hotmail.com
} 


\section{INTRODUÇÃO}

Conhecido como "Gameterapia" - a terapia através dos videogames - o uso dessa tecnologia acessível tem sido cada vez mais utilizada na reabilitação de crianças, adolescentes e idosos. Como bem nos assegura Alves (2020) no seu texto, a gameterapia é um recurso da fisioterapia a base de jogos de videogame, onde o paciente pode se exercitar de uma forma lúdica $\mathrm{e}$ agradável obtendo bons resultados de reabilitação.

O mais relevante, contudo, é constatar que a gameterapia possibilita uma interação do paciente com o equipamento, através de jogos digitais com sensores motores de movimento, promovendo uma reabilitação física e cognitiva. Sobre este aspecto, Rocha et al (2018) consideram que a realidade virtual (RV) como uma ferramenta em potencial para melhora de aspectos motivacionais e desempenho motor durante a reabilitação fisioterapêutica do paciente.

Muito se tem debatido sobre novas intervenções tecnológicas emergentes que possam atender essa demanda por ferramentas dinâmicas, lúdicas, como robôs complementares, bem como outros mecanismos de interação como voz ou tangível. Acerca desta discussão Nacher et al (2018), sinalizam para uma compreensão de que esses jogos podem contribuir para a melhora de alguns aspectos como o prazer, a socialização, e funções motoras; aumentar expressões emocionais; e reduzir a dor, ansiedade, angústia e estresse.

De certa forma, para muitos, o videogame é visto como uma brincadeira, para outros, uma competição, mas para os especialistas é considerado como um recurso inovador, eficiente e motivador no tratamento de reabilitação dos pacientes. Para Scapin et al (2018), além dessas possibilidades lúdicas de tratamento, o uso da $\mathrm{RV}$ promove uma redução no tempo de procedimentos dolorosos e na permanência nos leitos hospitalares.

Jurdi et al (2018) falam, resumidamente, que essas tecnologias de jogos apresentam funções voltadas para melhorar o prazer, a socialização, e funções motoras; aumentar expressões emocionais; e reduzir a dor, ansiedade, angústia e estresse. Tem-se nas discussões falas que nos remetem a uma compreensão de que a partir da revolução tecnológica, esses jogos espacialmente deslocaram-se da arena do mundo real para a web do mundo virtual. BHAT et al (2019). 


\section{MÉTODOLOGIA}

Trata-se de uma pesquisa caracterizada como revisão sistemática (de natureza qualitativa), realizada no sítio de compartilhamento de vídeos Youtube. A opção por este ambiente virtual adveio pelo simples fato de o mesmo sinalizar uma maior representatividade entre os usuários da Internet no que tange aos aspectos de compartilhamento, divulgação e visualização de vídeos. A versatilidade da revisão sistemática possibilita que sejam realizadas pesquisas pelas ópticas qualitativa e quantitativa e "[...] os resultados podem ser expostos na forma de conclusão, análise ou síntese" GOMES (2014, p. 398).

$$
\text { Quanto ao caminho }
$$

metodológico adotado para desenvolver esse estudo, pode ser percebido no direcionamento contemplado por meio de um protocolo de pesquisa, adaptado de instrumentos que foram validados por pesquisas semelhantes e composto dos seguintes elementos; tema da pesquisa; pergunta norteadora; objetivo geral; estratégias de busca; bancos de terminologias; avaliação crítica dos estudos; e apresentação dos resultados. Os indicadores analisados estão detalhados no Quadro 1.

Quadro 1 - Detalhamento das etapas da Revisão Sistemática.

\begin{tabular}{|c|c|c|c|c|}
\hline $\begin{array}{l}\text { ETA } \\
\text { PA }\end{array}$ & $\begin{array}{l}\text { TÓPICO } \\
\text { S DE } \\
\text { CADA } \\
\text { ETAPA }\end{array}$ & \multicolumn{3}{|c|}{ DETALHAMENTO DE CADA TÓPICO } \\
\hline \multirow[t]{10}{*}{$1^{\mathrm{a}}$} & Tema & \multicolumn{3}{|c|}{$\begin{array}{l}\text { Gameterapia como tecnologia assistiva: um registro descritivo em } \\
\text { ambiente virtual. }\end{array}$} \\
\hline & $\begin{array}{l}\text { Pergunta } \\
\text { norteado } \\
\text { ra }\end{array}$ & \multicolumn{3}{|c|}{ Qual a importância da gameterapia na tecnologia assistiva? } \\
\hline & $\begin{array}{l}\text { Objetivo } \\
\text { geral }\end{array}$ & \multicolumn{3}{|c|}{$\begin{array}{l}\text { Discutir cientificamente os vídeos compartilhados pelo sítio } \underline{\text { You Tube }} \\
\text { que tratam da gameterapia como tecnologia assistiva. }\end{array}$} \\
\hline & $\begin{array}{l}\text { Estratégi } \\
\text { as de } \\
\text { busca }\end{array}$ & \multicolumn{3}{|c|}{$\begin{array}{l}\text { Cruzamento de descritores por meio do operador booleano AND, OR; } \\
\text { Uso de aspas nos politermos (descritor com mais de um termo) para que } \\
\text { a varredura de vídeos contemplasse o termo exato; } \\
\text { Uso de descritores estruturados (codificação) no DECS ou MESH; } \\
\text { Uso de metadados (filtros) no You Tube }\end{array}$} \\
\hline & \multirow{3}{*}{$\begin{array}{l}\text { Bancos } \\
\text { de } \\
\text { terminol } \\
\text { ogias }\end{array}$} & Banco & \multicolumn{2}{|c|}{ Link } \\
\hline & & DeSC & \multicolumn{2}{|l|}{ http://decs.bvs.br/ } \\
\hline & & $\mathrm{MeSH}$ & \multicolumn{2}{|c|}{ https://www.ncbi.nlm.nih.gov/mesh } \\
\hline & \multirow{3}{*}{$\begin{array}{l}\text { Descrito } \\
\text { res livres } \\
\text { e } \\
\text { estrutur } \\
\text { ados }\end{array}$} & $\begin{array}{l}\text { Descrit } \\
\text { or }\end{array}$ & DeCS (Registro) & MeSH (IdentificadorÚnico) \\
\hline & & $\begin{array}{l}\text { gameter } \\
\text { apia }\end{array}$ & ------ & \\
\hline & & $\begin{array}{l}\text { Tecnolo } \\
\text { gia }\end{array}$ & $\begin{array}{c}----- \\
\end{array}$ & \\
\hline
\end{tabular}




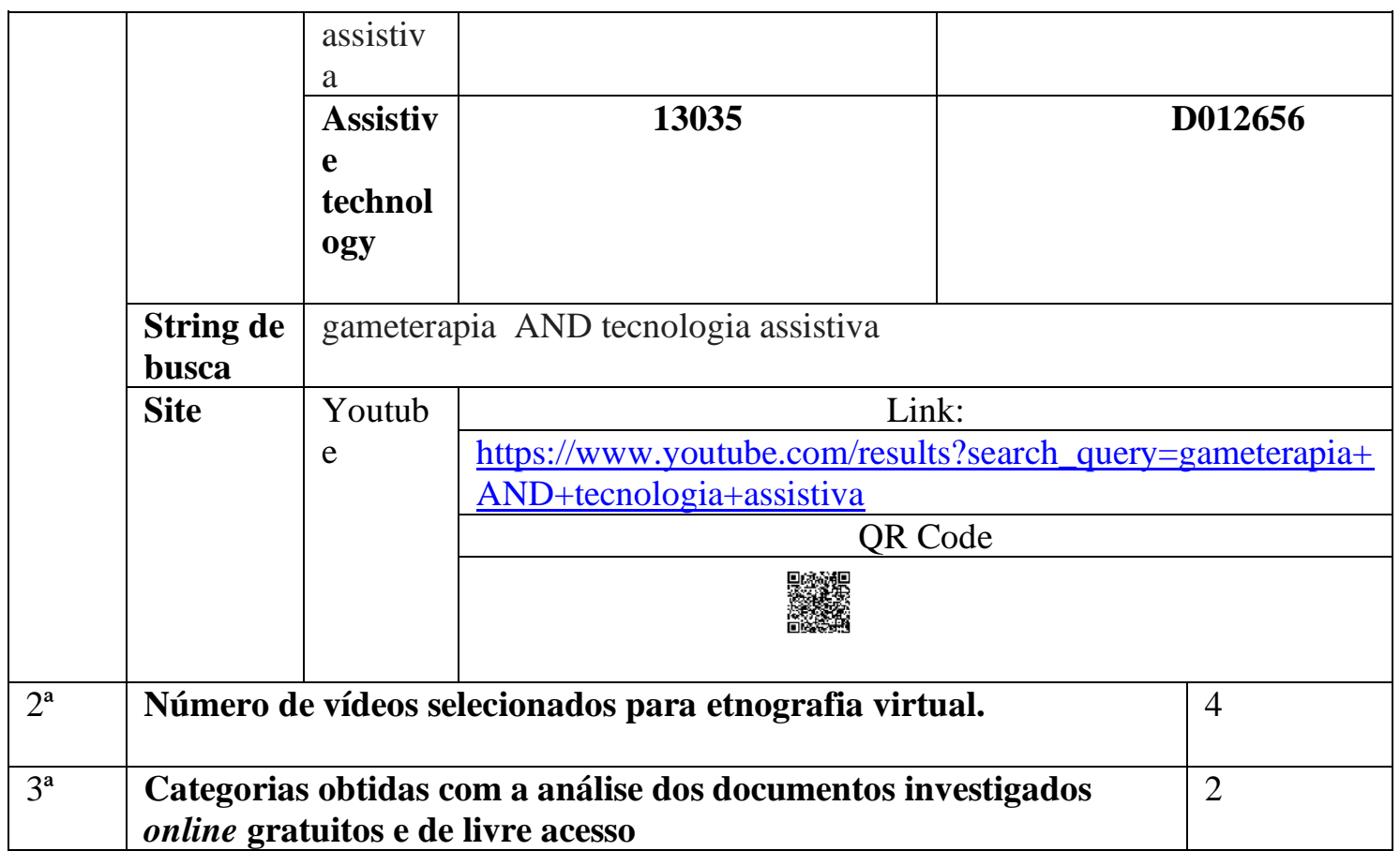

Fonte: elaborada pelos autores.

\begin{tabular}{|c|c|}
\hline $\begin{array}{l}\text { Indicador de } \\
\text { análise }\end{array}$ & Padronização \\
\hline Autor & $\begin{array}{l}\text { Responsável pela postagem do vídeo - colocar exatamente como aparece } \\
\text { e depois classificar (pessoa física órgão ou empresa/organização de } \\
\text { saúde) }\end{array}$ \\
\hline Tempo de duração & Indicado na timeline do vídeo (em minutos e segundos - nn'nn') \\
\hline Autor & $\begin{array}{l}\text { Responsável pela postagem do vídeo - colocar exatamente como aparece } \\
\text { e depois classificar (pessoa física, órgão ou empresa/organização de } \\
\text { saúde) }\end{array}$ \\
\hline Data da postagem & Indicado na descrição do vídeo (link Sobre abaixo do vídeo) \\
\hline $\begin{array}{l}\text { Total de } \\
\text { visualizações }\end{array}$ & Indicado abaixo do vídeo \\
\hline Categoria & $\begin{array}{l}\text { Segundo classificação do YouTube: indicado na descrição do vídeo } \\
\text { (link Sobre abaixo do vídeo, clica em Mostrar mais) }\end{array}$ \\
\hline Tema & Indicado na descrição do vídeo (link Sobre abaixo do vídeo) \\
\hline Total de inscritos & Indicado na descrição do vídeo (link Sobre abaixo do vídeo) \\
\hline Marcada & $\alpha$ \\
\hline Link do vídeo & \\
\hline Qrcode do vídeo & \\
\hline
\end{tabular}




\begin{tabular}{|c|c|}
\hline $\begin{array}{l}\text { Participantes do } \\
\text { vídeo }\end{array}$ & $\begin{array}{l}\text { Qual a importância da gameterapia na tecnologia assistiva? (indicar o } \\
\text { posicionamento) }\end{array}$ \\
\hline EQUIPE & $\begin{array}{l}\text { Indicar o posicionamento de todos os profissionais participantes no } \\
\text { vídeo. }\end{array}$ \\
\hline PACIENTE: & Indicar o posicionamento do paciente abordado? \\
\hline FAMÍLIA: & $\begin{array}{l}\text { Indicar o posicionamento e o grau parentesco dos familiares abordados } \\
\text { no vídeo. }\end{array}$ \\
\hline Escopo do vídeo & Resumo do conteúdo do vídeo de uma forma objetiva. \\
\hline $\begin{array}{l}\text { Paciente(s) } \\
\text { participante da(s) } \\
\text { atividade(s) }\end{array}$ & Informar a idade e o sexo (criança, adulto ou idoso) \\
\hline $\operatorname{Doença(s)}$ & Quais as enfermidades do paciente que participa do vídeo \\
\hline Local & Indicar a localização onde está sendo realizado o vídeo \\
\hline
\end{tabular}

\section{RESULTADOS}

Quadro 2 - Corresponde ao total de vídeos disponíveis no sítio do Youtube obtidos por string de busca.

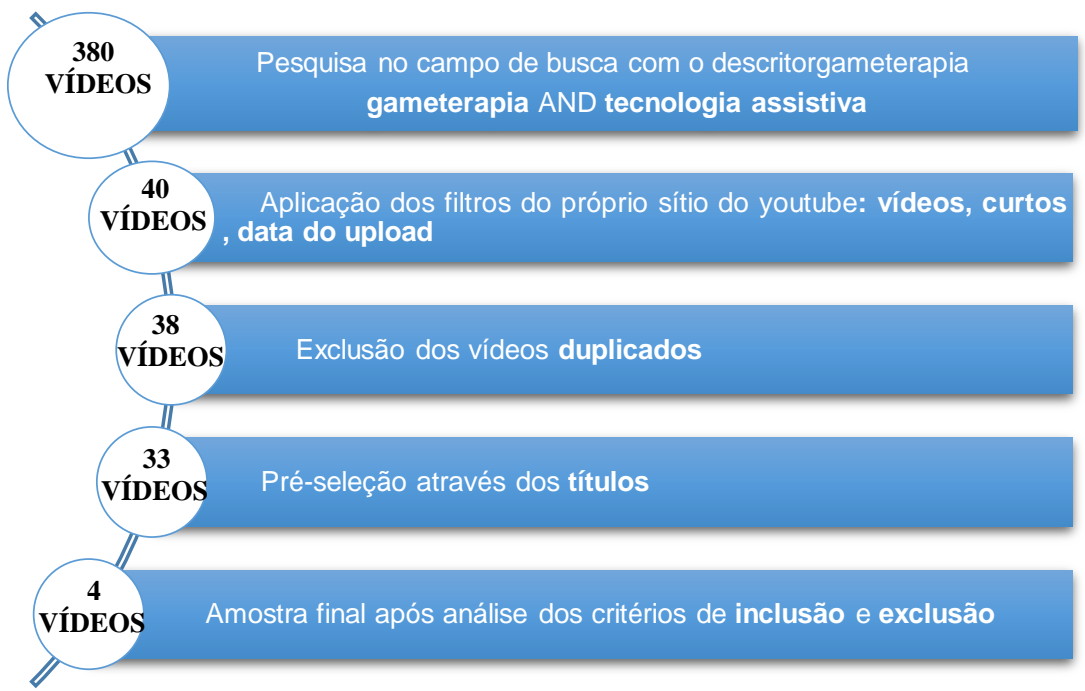

Fonte: elaborada pelos autores.

\section{VÍDEO 01}

\begin{tabular}{|l|l|}
\hline Autor & $\begin{array}{c}|c| \\
\text { UFPR TV } \\
\text { hosparvital de clínicas da Universidade Federal do Paraná (UFPR) }\end{array}$ \\
\hline Tempo de duração & Curto 3':49”' \\
\hline Data da postagem & 17 de julho de 2019 \\
\hline Total de visualizações & 144 \\
\hline Categoria & Educação \\
\hline Tema & GAMETERAPIA \\
\hline
\end{tabular}




\begin{tabular}{|c|c|}
\hline & $\begin{array}{l}\text { A interatividade dos jogos de videogame tem sido um grande recurso } \\
\text { para a reabilitação de pacientes do Ambulatório de Fisioterapia, do } \\
\text { Hospital de Clínicas da UFPR. A técnica é conhecida como } \\
\text { "Gameterapia" e tem auxiliado na melhora da força, do equilíbrio e } \\
\text { dos condicionamentos cardíaco e muscular dos pacientes. }\end{array}$ \\
\hline Total inscrito & 32,6 mil \\
\hline Marcada & ${ }_{8}$ \\
\hline Link & https://youtu.be/YyLiJY_XMOs \\
\hline QRCode & 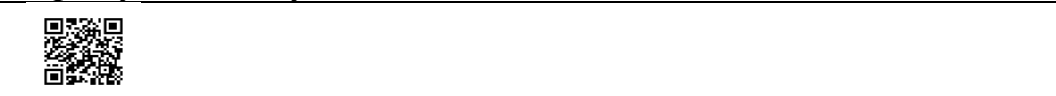 \\
\hline $\begin{array}{l}\text { PARTICIPANTE(S) } \\
\text { DO VÍDEO }\end{array}$ & O QUE É GAMETERAPIA? \\
\hline REPÓRTER(s): & $\begin{array}{l}\text { Repórter: a gameterapia uma solução dinâmica e recreativa que } \\
\text { atua na melhora da força muscular e dos condicionamentos } \\
\text { cardíaco e respiratório. }\end{array}$ \\
\hline $\begin{array}{l}\text { SUPERVISOR (es) } \\
\text { da(s) ATIVIDADE(s) }\end{array}$ & $\begin{array}{l}\text { Estudante de fisioterapia: "A Gameterapia então é a inserção } \\
\text { desses jogos virtuais com objetivo terapêutico então a gente faz esses } \\
\text { exercícios é com objetivo diferente tanto equilíbrio, a força . É } \\
\text { utilizando essa realidade que deixa mais é lúdico pro paciente } \\
\text { assim" }\end{array}$ \\
\hline PACIENTE(s): & $\begin{array}{l}\text { Idosa: “(..) tudo o que estou fazendo está melhorando está } \\
\text { melhorando por exemplo eu andar o equilíbrio a cabeça tudo } \\
\text { melhorou tudo de modo geral tudo né”. }\end{array}$ \\
\hline FAMÍLIA: & ------------------------- \\
\hline Escopo do vídeo & $\begin{array}{l}\text { Alunos do curso de fisioterapia desenvolveram uma pesquisa } \\
\text { técnico-científica (apoiado pela CAPES) chamada GAMETERAPIA } \\
\text { o método utiliza jogos virtuais para melhorar a qualidade de vida de } \\
\text { pessoas idosas. Equipe de pesquisadores que trabalham com } \\
\text { mulheres acima de } 65 \text { anos de idade e que apresentam uma condição } \\
\text { de pré-fragilidade(diminuição de força, perda de força, etc) . A } \\
\text { equipe é composta por: Professora Doutora do Departamento de } \\
\text { Fisioterapia UFPR; Doutoranda Educação Física UFPR; Estudante } \\
\text { de Fisioterapia UFPR; }\end{array}$ \\
\hline $\begin{array}{l}\text { Paciente(s) } \\
\text { participante da(s) } \\
\text { atividade(s) }\end{array}$ & Idosa de 86 anos de idade \\
\hline Doença(s) & $\begin{array}{l}\text { Fraqueza muscular e falta de equilíbrio .Fazendo a um ano ela } \\
\text { apresentou melhoras na sua mobilidade }\end{array}$ \\
\hline Local & $\begin{array}{l}\text { PARANÁ no Hospital universitário vinculado a rede hospitalar } \\
\text { Ebserh( Empresa Brasileira de Serviços Hospitalares - } \\
\text { https://www.ebserh.gov.br }\end{array}$ \\
\hline
\end{tabular}

\section{VÍDEO 2}

\begin{tabular}{|l|l|}
\hline Autor & R - Record News ES \\
\hline Tempo de duração & Curto 2':40” \\
\hline Data da postagem & 16 de agosto de 2015 \\
\hline Total de visualizações & 99 \\
\hline Categoria & Notícias e políticas \\
\hline Tema & GAMETERAPIA \\
\hline Marcada & \multicolumn{3}{|l|}{} \\
\hline
\end{tabular}




\begin{tabular}{|c|c|}
\hline Total inscritos & 22,8 mil \\
\hline Link & https://youtu.be/TmrqJW5uefQ \\
\hline QR Code & 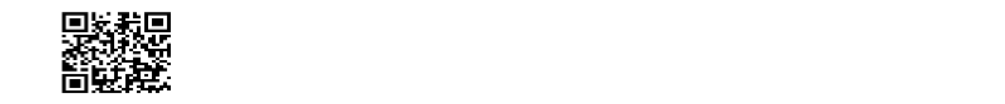 \\
\hline $\begin{array}{l}\text { PARTICIPANTE(S) DO } \\
\text { VÍDEO }\end{array}$ & O QUE É GAMETERAPIA? \\
\hline EQUIPE & $\begin{array}{l}\text { Repórter: uma nova terapia que conta com ajuda de jogos de } \\
\text { computador e os resultados são animadores } \\
\text { Professora: o tratamento com gametrapia ele tem como objetivo } \\
\text { melhorar a função motora desses indivíduos, melhorar o } \\
\text { equilíbrio favorecendo que a marcha desse indivíduo ela seja } \\
\text { melhor no seu dia-a-dia favorecendo uma melhor qualidade de } \\
\text { vida também para esse indivíduo. }\end{array}$ \\
\hline PACIENTE & É uma brincadeira que tem o seu RESULTADO POSITIVO \\
\hline \multicolumn{2}{|l|}{ FAMÍLIA } \\
\hline Escopo do vídeo & $\begin{array}{l}\text { Professora e estudantes de fisioterapeuta, desenvolvem um } \\
\text { programa que atende cerca de } 20 \text { pessoas por dia, tudo é feito de } \\
\text { forma lúdica utilizando o aparelho Kinect capta os movimentos } \\
\text { das pessoas por meio de sensores, os jogos estimulam o } \\
\text { desenvolvimento cognitivo, movimentos de braço e perna, e até } \\
\text { a caminhada. }\end{array}$ \\
\hline Paciente(s) & $\begin{array}{l}\text { Um idoso de } 60 \text { anos que sofre a } 28 \text { anos de mal de parkinson, } \\
\text { após um mês de tratamento sentiu já sentia melhoras. }\end{array}$ \\
\hline Doença(s) & $\begin{array}{l}\text { Mal de parkson (o idoso estava com mãos trêmulas e dificuldade } \\
\text { pra caminhar) }\end{array}$ \\
\hline Local & $\begin{array}{l}\text { É desenvolvido na faculdade Vitória (EMESCAM) a um ano, } \\
\text { atendimento gratuito }\end{array}$ \\
\hline
\end{tabular}

\section{VÍDEO 3}

\begin{tabular}{|c|c|}
\hline Autor & JORNAL PARANAÍBA \\
\hline Tempo de duração & Curto 3':56" \\
\hline Data da postagem & 8 de fevereiro de 2019 \\
\hline Total de visualizações & 238 \\
\hline Categoria & Notícias e políticas \\
\hline Tema & $\begin{array}{l}\text { JORNAL PARANAÍBA - Pesquisadores criam jogo para } \\
\text { reabilitação de braços após avc }\end{array}$ \\
\hline Total inscrito & $222 \mathrm{mil}$ \\
\hline Marcada & $\mathrm{O}_{8}$ \\
\hline Link & https://youtu.be/waeu-7U-tAk \\
\hline QR Code & 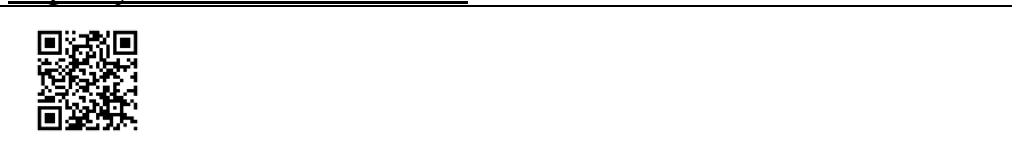 \\
\hline $\begin{array}{l}\text { PARTICIPANTE(S) DO } \\
\text { VÍDEO }\end{array}$ & O QUE É GAMETERAPIA? \\
\hline EQUIPE & $\begin{array}{l}\text { Repórter: é uma terapia experimental; A parte lúdica do game } \\
\text { prende a atenção do paciente e aí ele se movimenta e se esforça } \\
\text { até mesmo sem perceber } \\
\text { Pesquisador em computação: o bracelete que o paciente } \\
\text { controla ele tem uma certa precisão que pode ser de acordo com }\end{array}$ \\
\hline
\end{tabular}




\begin{tabular}{|l|l|}
\hline & $\begin{array}{l}\text { o paciente se ele consegue aumentar o ângulo do braço ou não o } \\
\text { terapeuta vai configurar esse movimento e ele vai desenvolvesse- } \\
\text { se ao longo do percurso. } \\
\text { Pesquisadora em engenharia biomédica: O paciente não está } \\
\text { indo para uma clínica de reabilitação, ele vai vir jogar pra fazer } \\
\text { alguma coisa lúdica, ele vai ver que o braço dele no caso desse } \\
\text { jogo é um animal e ele mexe, ele voa, ele faz coisas que ele não } \\
\text { tinha ideia que ele poderia voltar a fazer; }\end{array}$ \\
\hline PACIENTE & $\begin{array}{l}\text { Idoso: Senti melhor mesmo, o meu braço soltou mais e parece } \\
\text { que ele trabalhou os músculos, os músculos ficam muito parado } \\
\text { né. }\end{array}$ \\
\hline FAMÍLIA & $\begin{array}{l}\text { PESQUISADORES da universidade federal de Uberlândia } \\
\text { (UFU) desenvolveram uma terapia para pacientes com derrame } \\
\text { cerebral. O diferencial é que os exercícios de reabilitação são } \\
\text { feitos através de um jogo eletrônico. Diferente da reabilitação que } \\
\text { conhecemos, a pessoa recupera os movimentos se divertindo. }\end{array}$ \\
\hline Descrição do vídeo & Um idoso \\
\hline Paciente(s) & AVC \\
\hline Doença(s) & UBERLÂNDIA \\
\hline LOCAL &
\end{tabular}

\section{VÍDEO 4}

\begin{tabular}{|c|c|}
\hline Autor & \\
\hline Tempo de duração & Curto $1 ': 56 "$ \\
\hline Data da postagem & 23 de março de 2017 \\
\hline Total de visualizações & 1.576 \\
\hline Categoria & Ciências e tecnologia \\
\hline Tema & BIOMOVI - DEMONSTRAÇÃO BIOSPACE \\
\hline Total inscrito & 728 \\
\hline Marcada & $\mathcal{O}_{8}$ \\
\hline Link & https://youtu.be/0fFGuGBApO0 \\
\hline QR Code & 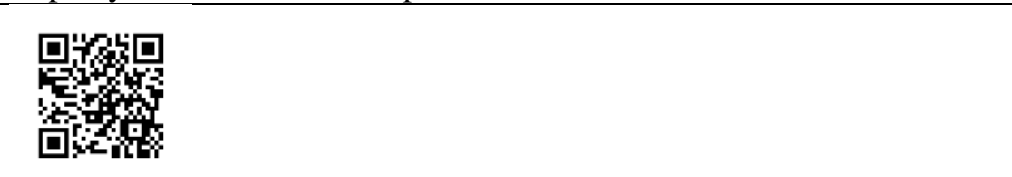 \\
\hline $\begin{array}{l}\text { PARTICIPANTE(S) DO } \\
\text { VIIDEO }\end{array}$ & O QUE É GAMETERAPIA? \\
\hline EQUIPE & $\begin{array}{l}\text { Ele é uma FORMA INTELIGENTE de fazer com que o usuário se } \\
\text { sinta imerso em um cenário virtual e substitua o "Joystick" pela } \\
\text { contração muscular, fazendo com que os objetos do jogo ganhem } \\
\text { movimento e ao final de uma sessão do game, seja possível ver os pontos } \\
\text { que foram obtidos, assim como observar de forma gráfica como foi a } \\
\text { atividade muscular produzida em cada momento do jogo. }\end{array}$ \\
\hline PACIENTE & 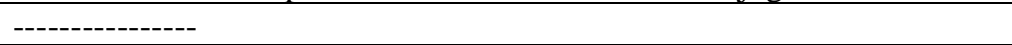 \\
\hline FAMÍLIA & 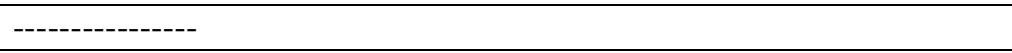 \\
\hline $\begin{array}{lr}\text { Supervisor(es) } & \text { que estavam } \\
\text { acompanhado } & \mathrm{a}(\mathrm{s}) \\
\text { atividade(s) } & \\
\end{array}$ & ----- \\
\hline Paciente(s) & ----- \\
\hline Doença(s) & \\
\hline LOCAL & Porto Alegre - Rio Grande do Sul \\
\hline
\end{tabular}


Quadro 3- Quadro matricial da primeira categoria.

\begin{tabular}{|c|c|c|c|}
\hline \multicolumn{4}{|c|}{$\begin{array}{l}\text { Definição: Gameterapia é uma técnica que utiliza jogos virtuais com objetivo terapêutico para melhorar a qualidade de vida das } \\
\text { pessoas. }\end{array}$} \\
\hline PALAVRA & FREQUÊNCIA & PARTICIPANTE/PROPOSIÇÃO & $\mathbf{V} / \mathbf{F}$ \\
\hline \multirow[t]{2}{*}{ GAMETERAPIA } & 3 & $\begin{array}{l}\text { V1- o hospital de clínicas da UFPR utiliza jogos virtuais no tratamento de } \\
\text { doenças cardiorespiratórias neurológicas e músculo-esqueléticas pacientes } \\
\text { com artrite fibromialgia escleroses e outras doenças são beneficiados com a } \\
\text { GAMETERAPIA uma solução dinâmica e recreativa que atua na melhora da } \\
\text { força muscular e dos condicionamentos cardíaco e respiratório. (...).A } \\
\text { GAMETERAPIA tem se intensificado na prática fisioterapêutica porque tem } \\
\text { bons resultados e boa aderência dos pacientes cada um dos jogos simula } \\
\text { movimentos reais para atingir diferentes objetivos. (...) GAMETERAPIA então } \\
\text { é a inserção desses jogos virtuais com objetivo terapêutico }\end{array}$ & $\mathbf{V}$ \\
\hline & 2 & $\begin{array}{l}\text { V2- A GAMETERAPIA é recente no Brasil estar no país apenas dois anos e já } \\
\text { um sucesso no Espírito Santo, a terapia é um recurso muito usado } \\
\text { principalmente para quem sofre doença de Parkinson. A idéia é superar os } \\
\text { desafios. Tratamento com GAMETRAPIA ele tem como objetivo melhorar a } \\
\text { função motora desses indivíduos, melhorar o equilíbrio favorecendo que a } \\
\text { marcha desse indivíduo ela seja melhor no seu dia-a-dia favorecendo uma } \\
\text { melhor qualidade de vida também para esse indivíduo }\end{array}$ & $\mathbf{V}$ \\
\hline DINÂMICA & 1 & $\begin{array}{l}\text { V1- (...) pacientes com artrite fibromialgia escleroses e outras doenças são } \\
\text { beneficiados com a gameterapia uma solução DINÂMICA e recreativa que } \\
\text { atua na melhora da força muscular e dos condicionamentos cardíaco e } \\
\text { respiratório }\end{array}$ & $\mathbf{V}$ \\
\hline RECREATIVA & 1 & $\begin{array}{l}\text { V1 - (...) pacientes com artrite fibromialgia escleroses e outras doenças são } \\
\text { beneficiados com a GAMETERAPIA uma solução dinâmica e } \underline{\mathbf{R E C R E A T I V A}} \\
\text { que atua na melhora da força muscular e dos condicionamentos cardíaco e } \\
\text { respiratório }\end{array}$ & $\mathbf{V}$ \\
\hline \multicolumn{4}{|c|}{$\begin{array}{l}\text { Definição: Videogames - atuais games com sensores que permitem comandar um jogo com o corpo, concebido como um } \\
\text { complemento para vários tratamentos além da reabilitação motora, da coordenação motora, a melhora do equilíbrio - ele age } \\
\text { também na cognição, melhora a memória, a atençâo, a concentração influenciando na motivação e na autoestima dos pacientes }\end{array}$} \\
\hline PALAVRA & FREQUÊNCIA & $\begin{array}{l}\text { PARTICIPANTE/PROPOSIÇÃO } \\
\end{array}$ & $\mathbf{V} / \mathbf{F}$ \\
\hline VÍDEOGAME & 1 & $\begin{array}{l}\text { V3 "um jogo eletrônico, (...) pouco diferente do que estamos acostumados não } \\
\text { tem controle de VIDEOGAME, mas sim um bracelete com sensor e não é só } \\
\text { por diversão esta é uma terapia experimental criada por pesquisadores da } \\
\text { universidade de Uberlândia para pessoas que sofreram AV e ficaram com } \\
\text { sequela em um dos braços. }\end{array}$ & $\mathbf{F}$ \\
\hline \multirow[t]{2}{*}{ MÉTODO } & 2 & $\begin{array}{l}\text { V1 - gameterapia o } \underline{\text { MÉTODO }} \text { utiliza jogos virtuais para melhorar a qualidade } \\
\text { de vida de pessoas idosas (...), com a técnica aplicada (...) a mobilidade da } \\
\text { paciente melhorou (....). há um ano participando da iniciativa a paciente já sente } \\
\text { os beneficios da gameterapia (...)”. }\end{array}$ & $\mathbf{V}$ \\
\hline & & $\begin{array}{l}\text { V3 “um jogo eletrônico, (...) é uma terapia experimental (...). O projeto vem } \\
\text { sendo desenvolvido a quatro anos e após todos os ajustes foi possível começar } \\
\text { a testar em pacientes. O seu Antônio é um dos cinco participantes ele até já } \\
\text { passou por outros MÉTODOS convencionais de recuperação, mas ele garante } \\
\text { esse é o mais inovador. }\end{array}$ & $\mathbf{V}$ \\
\hline INOVADOR & 1 & $\begin{array}{l}\text { V3 “um jogo eletrônico,(...) é uma terapia experimental (...). O seu Antônio é } \\
\text { um dos cinco participantes ele até já passou por outros métodos convencionais } \\
\text { de recuperação, mas ele garante esse é o mais INOVADOR.(...) A parte lúdica } \\
\text { do game prende a atenção do paciente e aí ele se movimenta e se esforça até } \\
\text { mesmo sem perceber. }\end{array}$ & $\mathbf{V}$ \\
\hline JOGO & & $\begin{array}{l}\text { V 4- O BioMovi é o primeiro GAME DE REALIDADE VIRTUAL que utiliza a } \\
\text { atividade produzida pelos músculos para dar movimento aos jogos, auxiliando } \\
\text { na reabilitação ou melhoria de performance física dos usuários. Ele é uma } \\
\text { forma inteligente de fazer com que o usuário se sinta imerso em um cenário } \\
\text { virtual e substitua o "Joystick" pela contração muscular, fazendo com que os } \\
\text { objetos do jogo ganhem movimento e ao final de uma sessão do game, seja } \\
\text { possivel ver os pontos que foram obtidos, assim como observar de forma gráfica } \\
\text { como foi a atividade muscular produzida em cada momento do jogo. }\end{array}$ & $\mathbf{V}$ \\
\hline
\end{tabular}


Quadro 4 - Descrição dos documentos (vídeos) de acordo com os critérios de inclusão.

\begin{tabular}{|c|c|c|c|}
\hline Vídeos & Tema & $\begin{array}{c}\text { Data } \\
\text { do } \\
\text { Upload }\end{array}$ & $\begin{array}{l}\text { Fala do(s) participante(s) que } \\
\text { atendem a pergunta norteadora }\end{array}$ \\
\hline $\begin{array}{l}\text { Vídeo } \\
1\end{array}$ & $\begin{array}{l}\text { GAMETERAPIA } \\
\text { A interatividade dos jogos de } \\
\text { videogame tem sido um grande } \\
\text { recurso para a reabilitação de } \\
\text { pacientes do Ambulatório de } \\
\text { Fisioterapia, do Hospital de } \\
\text { Clínicas da UFPR. A técnica é } \\
\text { conhecida como "Gameterapia" } \\
\text { e tem auxiliado na melhora da } \\
\text { força, do equilíbrio e do } \\
\text { condicionamentos cardíaco e } \\
\text { muscular dos pacientes. }\end{array}$ & 2019 & $\begin{array}{l}\text { Repórter: "a gameterapia uma } \\
\text { solução dinâmica e recreativa que atua } \\
\text { na melhora da força muscular e dos } \\
\text { condicionamentos cardiaco } \\
\text { respiratório." } \\
\text { Estudante } \\
\text { Gameterapia então é a inserção desses } \\
\text { jogos virtuais com objetivo terapêutico } \\
\text { então a gente faz esses exercícios é com } \\
\text { objetivo diferente tanto equilíbrio, a } \\
\text { força. É utilizando essa realidade que } \\
\text { deixa mais é lúdico pro paciente assim" } \\
\text { Idosa: "tudo o que estou fazendo está } \\
\text { melhorando está melhorando por } \\
\text { exemplo eu andar o equilíbrio a cabeça } \\
\text { tudo melhorou tudo de modo geral tudo } \\
\text { né" }\end{array}$ \\
\hline $\begin{array}{l}\text { Vídeo } \\
2\end{array}$ & $\begin{array}{l}\text { GAMETERAPIA - Record } \\
\text { News ES }\end{array}$ & 2015 & $\begin{array}{l}\text { Repórter: uma nova terapia que conta } \\
\text { com ajuda de jogos de computador e os } \\
\text { resultados são animadores } \\
\text { Professora: o tratamento com } \\
\text { gametrapia ele tem como objetivo } \\
\text { melhorar a função motora desses } \\
\text { indivíduos, melhorar o equilíbrio } \\
\text { favorecendo que a marcha desse } \\
\text { indivíduo ela seja melhor no seu dia-a- } \\
\text { dia favorecendo uma melhor qualidade } \\
\text { de vida também para esse indivíduo. }\end{array}$ \\
\hline $\begin{array}{l}\text { Vídeo } \\
3\end{array}$ & $\begin{array}{l}\text { JORNAL PARANAÍBA- } \\
\text { Pesquisadores criam jogo } \\
\text { para reabilitação de braços } \\
\text { após AVC } \\
\text { Pesquisadores da UFU } \\
\text { desenvolveram uma terapia para } \\
\text { pacientes com derrame cerebral. } \\
\text { O diferencial é que os exercícios } \\
\text { de reabilitação são feitos através } \\
\text { de um jogo eletrônico. Diferente } \\
\text { da reabilitação que conhecemos, } \\
\text { a pessoa recupera os } \\
\text { movimentos se divertindo. }\end{array}$ & 2019 & $\begin{array}{l}\text { Repórter: é uma terapia experimental; } \\
\text { A parte lúdica do game prende a } \\
\text { atenção do paciente e aí ele se } \\
\text { movimenta e se esforça até mesmo sem } \\
\text { perceber } \\
\text { Pesquisador em computação: o } \\
\text { bracelete que o paciente controla ele } \\
\text { tem uma certa precisão que pode ser de } \\
\text { acordo com o paciente se ele consegue } \\
\text { aumentar o ângulo do braço ou não o } \\
\text { terapeuta vai configurar esse } \\
\text { movimento e ele vai desenvolvesse-se } \\
\text { ao longo do percurso. } \\
\text { Pesquisadora em engenharia } \\
\text { biomédica: O paciente não está indo } \\
\text { para uma clínica de reabilitação, ele } \\
\text { vai vir jogar pra fazer alguma coisa } \\
\text { lúdica, ele vai ver que o braço dele no } \\
\text { caso desse jogo é um animal e ele mexe } \\
\text { ele voa, ele faz coisas que ele não } \\
\text { tinha ideia que ele poderia voltar a } \\
\text { fazer; }\end{array}$ \\
\hline
\end{tabular}




\begin{tabular}{|l|l|l|l|}
\hline $\begin{array}{l}\text { Vídeo } \\
4\end{array}$ & $\begin{array}{l}\text { BIOMOVI } \\
\text { DEMONSTRAÇÃO } \\
\text { BIOSPACE }\end{array}$ & 2017 & $\begin{array}{l}\text { Ele é uma FORMA INTELIGENTE } \\
\text { de fazer com que o usuário se sinta } \\
\text { imerso em um cenário virtual e } \\
\text { substitua o "Joystick" pela contração } \\
\text { muscular, fazendo com que os objetos } \\
\text { do jogo ganhem movimento e ao final } \\
\text { de uma sessão do game, seja possível } \\
\text { ver os pontos que foram obtidos, assim } \\
\text { como observar de forma gráfica como } \\
\text { foi a atividade muscular produzida em } \\
\text { cada momento do jogo. }\end{array}$ \\
\hline
\end{tabular}

Fonte: Site online do Youtube, 2019.

O escopo das falas dos participantes dos vídeos foi analisado por meio da frequência de palavras, que gerou a nuvem de palavras (Figura 1)

Figura 1 - Nuvem de palavras

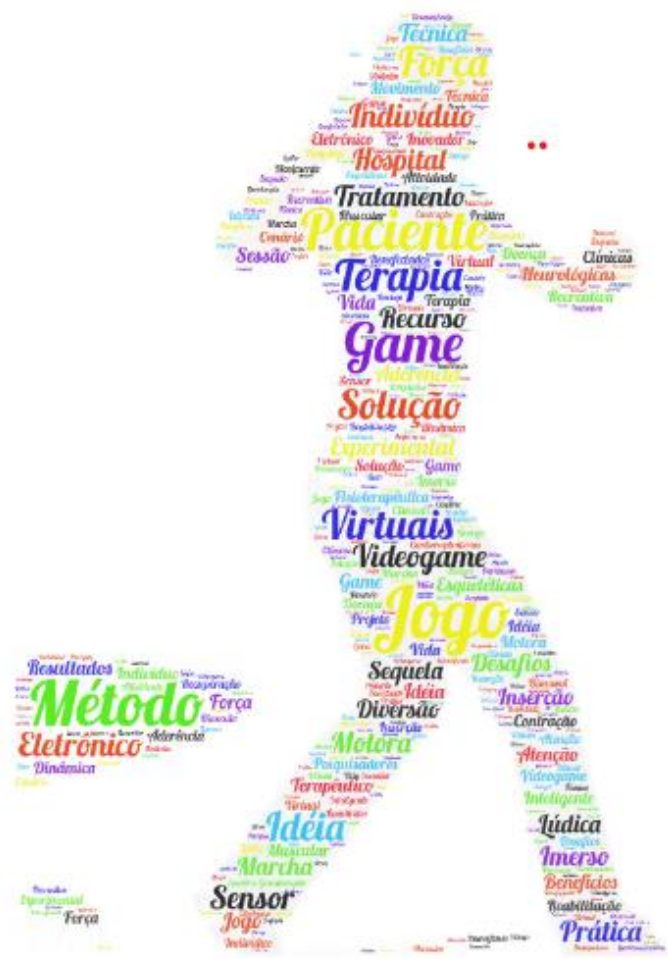

Fonte: Elaboda pelos autores.

Por meio da Figura 1, foi possível observar que as palavras em evidência na nuvem pertencem as categorias desenvolvidas a partir da análise de criada na Plataforma online WordArt. Esta ferramenta reúne e sistematiza graficamente as palavras-chave destacando-as as mais frequentes.

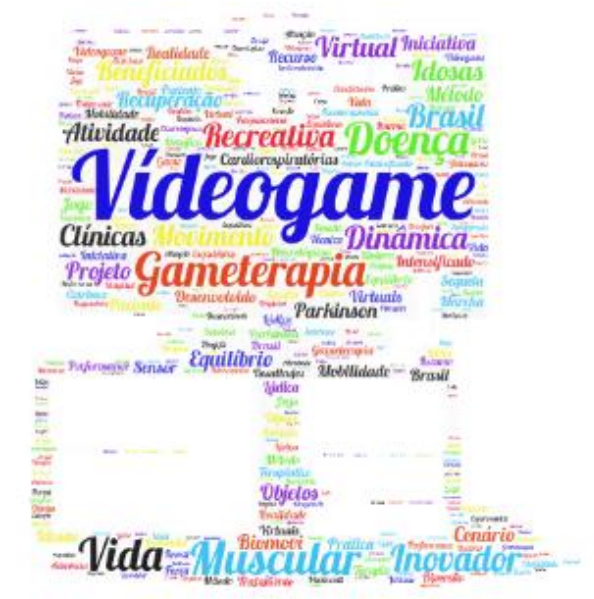

conteúdo de Bardin. Todas as categorias derivam da sua frequência (Tabela 1), que diz respeito ao seu quadro referencial. Em consonância ao objetivo 
deste trabalho, optou-se por descrever as palavras que apresentaram frequência total no texto e, a partir de seus sentidos nos campos textuais, tinham maior relevância para as representações sociais sobre a gameterapia como tecnologia assistiva, como apresentado na Figura1.

Tabela 1. Frequência das palavras dos participantes presentes nos vídeos postados no sítio do Youtube.

\begin{tabular}{l|c|l}
\hline \multicolumn{1}{c|}{ PALAVRAS } & FREQUÊNCIA & \multicolumn{1}{c}{ CATEGORIAS } \\
\hline Gameterapia & 5 & \multirow{2}{*}{ Gameterapia uma solução Dinâmica e Recreativa } \\
\cline { 1 - 2 } Dinâmica & 1 & \\
\hline Recreativa & 1 & \multirow{2}{*}{ Vídeogame um método inovador. } \\
\hline Vídeogame & 1 & \\
\hline Método & 2 & \\
\hline Inovador & 1 & \\
\hline
\end{tabular}

Fonte: elaborada pelos autores.

\section{DISCUSSÃO}

A partir da leitura sistemática do conteúdo dos vídeos, duas categorias foram estruturadas para análise:

\section{Gameterapia uma solução dinâmica e recreativa}

Os especialistas alertam que o tratamento de reabilitação deve seguir um protocolo de atendimento ao paciente no que tange às práticas específicas voltadas para esse atendimento, mas, são claros e otimistas - quando o assunto em pauta é a Gameterapia a qual é vista como recurso fisioterapêutico e motivador em que o paciente se sente estimulado a realizar os movimentos apresentados pelos videogames. Sobre este aspecto Araújo et al (2020), afirmam o quão é relevante instituir um protocolo de avaliação que contemple diversos benefícios da terapia de RV, sendo esta capaz de mensurar ganhos e ter resultados consistentes na aplicação instrumentos e testes.

Sendo percebida como "uma solução dinâmica e recreativa (Vídeo 1) que atua na melhora da força muscular e dos condicionamentos cardíaco e respiratório. (...). A gameterapia tem se intensificado na prática fisioterapêutica porque tem bons resultados e boa aderência dos pacientes cada um dos jogos simula movimentos reais para atingir diferentes objetivos. Então, é fato que a sociedade vive numa era digital em rápida evolução na qual ambos os riscos e perspectivas on-line são postos de forma abundantes.

De acordo com Glover et al (2018), é fundamental que os profissionais de saúde mental apreendam e gerenciem os riscos, concomitantemente à medida em que 
adotam os benefícios potenciais e as possibilidades de tratamento que $\mathrm{o}$ mundo digital beneficia aos pacientes.

Nesse contexto de possibilidades interventivas, apresenta-se o BioMovi (Vídeo 4) como sendo o primeiro game de RV que faz uso da atividade produzida pelos músculos para dar movimento aos jogos, auxiliando na reabilitação ou melhoria de performance física dos usuários. Papanastasiou, at al (2017), a cerca a desta questão comentam que ao projetar videogames, cientistas do comportamento e outros profissionais ilustram as mudanças positivas relacionadas à saúde que eles fornecem e as propriedades envolventes da interatividade, combinadas com a tecnologia de mudança de comportamento que eles fundem.

De certa forma, tais instrumentos podem ser vistos como complementos úteis à terapia e devem ser considerados como uma ferramenta de reabilitação eficaz nesse processo.

Pode-se considerar a Gameterapia como sendo uma técnica que utiliza jogos virtuais com fins terapêuticos para melhorar a qualidade de vida das pessoas. Ultimamente, vem se intensificando no campo da prática fisioterapêutica, justifica-se, não só pelo alcance de bons resultados como também pela aderência expressiva dos pacientes a essa técnica.

Acerca da relevância desse método, Diasa (2016) realça que a gameterapia, exclusivamente através desses jogos virtuais, verifica-se no seu escopo determinadas funções motoras próprias ao videogame e estimulantes para o aprimoramento da função motora grossa. Muito além da superação da recuperação do movimento, a ideia é superar os desafios (Vídeo 2), melhorar o equilíbrio e/ou outras enfermidades, esse tratamento desenha-se na possibilidade de favorecer a uma melhor qualidade de vida para o paciente.

Existem diversas possibilidades de jogos virtuais que, através de sensores de movimento controlam o movimento do corpo e promovendo uma interação entre paciente e equipamento de forma a conduzi-lo a execução do exercício sem nem se perceber que o estar fazendo. A ludicidade presente no game leva-o ao mundo da RV e, nesse cenário suas limitações parecem não existir mais.

\section{Vídeogame um método inovador}

Surgiram no final da década de 70, os videogames, estes configuram-se como uma modalidade de RV, finalidade esta percebida por promover ao paciente adentrar em espaços virtuais, manipular objetos inseridos nesse ambiente como 
também poder transitar nesse cenário realizando movimentos que fora dele não seria possível, viabilizam oportunidades de interações únicas CORRÊA et al (2011). Acerca desta discussão, Carbonera et al (2016), citam que nesse ambiente o prazer e a aderência ambos devem ser considerados ao avaliar ou escolher uma modalidade de exercício com videogames interativos.

Apesar dessa perspectiva de surgimento ser bastante antiga, atualmente esse recurso tecnológico vem ganhando adeptos cada vez mais. Surpreendentemente, o efeito estimulador que provoca nos pacientes transcende as dores pertinentes a enfermidade causada pelo trauma acometido pela doença. Suenderhauf et al (2016) reforçam a ideia de que os videogames devem ser considerados como terapêuticos e formas de intervenções.

\section{CONCLUSÃO}

O tratamento com gameterapia sinaliza para uma complementação no contexto da terapia convencional incrementado uma forma mais dinâmica, recreativa e interativa para o paciente no tocante à realização dos exercícios. Traz no seu escopo uma possibilidade de adesão a essa prática de forma a
Sendo assim, o método utilizado com videogames, conhecido como Gameterapia, não atingi apenas os pacientes que apresentam enfermidades, o seu raio de ludicidade contamina também àqueles que estão acompanhando o processo de tratamento. A interação acaba acontecendo com os outros familiares que se sentem seduzidos com game e com isso passar ser uma peça também importante nesse contexto.

Por ser considerada uma terapia experimental (Vídeo 3), pode-se concluir que, tais videogames são um complemento útil à terapia e devem ser considerados como uma ferramenta de reabilitação dentro do ambiente hospitalar e para uso domiciliar em pacientes em processo de recuperação terapêutica. KAWADA et al (2015).

engendrar resultados positivos e satisfatórios apontados na melhora, não só dos aspectos motores, equilíbrio, concentração, coordenação motora, mas principalmente no desenho da usa autoestima e motivação. Esses ganhos estão presentes também na redução de permanência do leito e na aquisição de uma boa qualidade vida significativa. 


\section{REFERÊNCIAS}

ALVES, B. Colaboração para

VivaBem, 01 de maio de 2020.

Disponível em:

<https://www.uol.com.br/vivabem/notic ias/redacao/2020/05/01/idoso-tambemjoga-videogame-e-e-tratamento-desaude-conheca-a-gameterapia.htm >acesso em:11 março 2021.

ARAÚJO et al. Non-pharmacological therapeutic strategy options for patients with dementia based on cognitive function-A Bayesian network metaanalysis of randomized controlled trials ,2020.

BATISTA, J. S. et al. Reabilitação de idosos alterações cognitivas através do Nintendo wii. Revista Brasileira de Ciência do Envelhecimento Humano, Passo Fundo, v.9, n.2, p.293-299, 2012.

BHAT, O. J.; PRAKASH. S. K. Game of thorns: Modern day opium, 2019.

CORRÊA, A. G. D. et al. Realidade virtual e jogos eletrônicos: uma proposta para deficientes. In; MONTEIRO, C.B.M. (Org.). Realidade virtual na Paralisia Cerebral. São Paulo: Plêiade, 2011.p.93-108.

CARBONERA, R. P.; Vendrusculo, F. M.; DONADIO, M. V. F. Physiological responses during exercise with video games in patients with cystic fibrosis: A systematic review, 2016

DIASA et al. As contribuições da gameterapia no desempenho motor de indivíduo com paralisia cerebral1, 2016.

GOMES, I. S.; CAMINHA, I. O. Guia para estudos de revisão sistemática: uma opção metodológica para as
Ciências do Movimento Humano. Movimento, v. 20, n. 1, p. 395-411, jan./mar. 2014.

GLOVER, J. PHD, Sandra L. Fritsch, MD, MSEd*. \#KidsAnxiety and Social Media: A Review,2018.

JURDI et al. A systematic review of game technologies for pediatric patients, 2018.

NACHER et al. A systematic review of game technologies for pediatric patients, 2018.

PAPANASTASIOU et al. PatientCentric ICTs based Healthcare for students with learning, physical and/ or sensory disabilities, 2017.

KAWADA, et al. A Pilot Prospective Randomized Control TrialComparing Exercises Using Videogame Therapy toStandard Physical Therapy: 6 Months FollowUp, 2015.

ROCHA et al. El efecto de la intervención con videojuego activo sobre el auto concepto, el equilibrio, el desempeño motor y el éxito adaptativo de niños con parálisis cerebral: el estudio preliminar, 2018.

SCAPIN et al. Além dessas possibilidades lúdicas de tratamento, o uso da RV promove uma redução no tempo de procedimentos dolorosos e na permanência nos leitos hospitalares, 2018.

SUENDERHAUF, C;. WALTER, A. L. C. UNDINE, E. BORGWARDT Lang, Stefan. Counter striking psychosis: Commercial video games as potential treatment in schizophrenia? A systematic review of neuroimaging studies, 2016. 